\title{
Plaguicidas para el manejo del pulgón amarillo (Melanaphis sacchari, Zehnter), en sorgo (Sorghum bicolor L. Moench), en Nicaragua
}

\section{Pesticides for the management of the yellow aphid (Melanaphis sacchari, Zehnter), in sorghum (Sorghum bicolor L. Moench), in Nicaragua}

\section{Edgardo Jiménez-Martínez ${ }^{1}$, Nelson Reyes Canales ${ }^{2}$, Lenard Rivas Espinoza ${ }^{2}$}

${ }^{1}$ PhD en Entomología, ORCID: https://orcid.org/0000-0003-1086-7380, edgardo.jimenez@ci.una.edu.ni, (505) 2263-2609

${ }^{2}$ Ingeniero Agrónomo.

Universidad Nacional Agraria

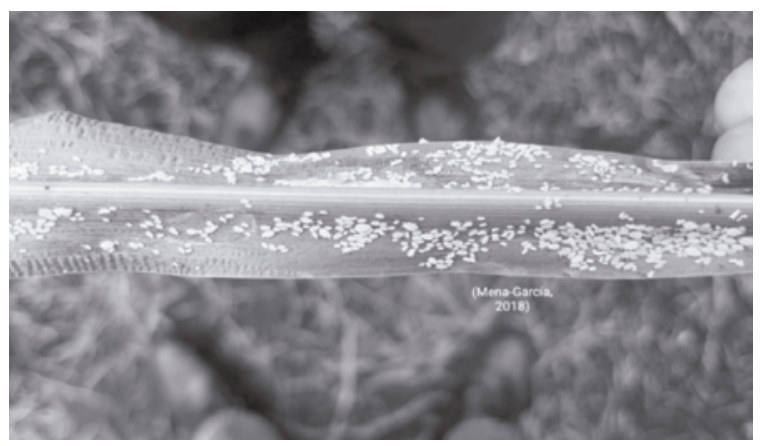

\section{RESUMEN}

Con el objetivo de evaluar insecticidas químicos, biológicos y botánicos para el manejo del pulgón amarillo del sorgo, se realizó un estudio en la finca "El Plantel" en el período comprendido de agosto a octubre del año 2017. Las alternativas evaluadas fueron: Engeo, Imidacloprid, Beauveria bassiana, Metharizium anisopliae y Chile+ajo+jabón en comparación con el Testigo que fue solamente aplicación de agua. Las variables evaluadas fueron: número de ninfas de $M$. sacchari por planta, de adultos de M. sacchari por planta, de alados de $M$. sacchari planta, además de algunas variables económicas como el rendimiento en $\mathrm{kg} \mathrm{ha}^{-1}$ por tratamiento evaluado, análisis de presupuesto parcial, análisis de dominancia y tasa de retorno marginal. Los resultados obtenidos en el estudio determinan que los tratamientos Engeo e Imidacloprid fueron los que presentaron el mejor control de ninfas, adultos y alados de M. sacchari, los mejores rendimientos comerciales lo obtuvieron los tratamientos Engeo e Imidacloprid, las mejores tasas de retorno marginal fueron obtenidas en el tratamiento Imidacloprid.

Palabras clave: insecticidas, plagas, control.
Recibido: 12 de julio del 2019 Aceptado: 3 de septiembre del 2019

\begin{abstract}
With the objective of evaluating chemical, biological and botanical insecticides, for the management of the yellow sorghum aphid, a study was carried out on the "El Plantel" farm in the period from August to October of the year 2017. The alternatives evaluated were: Engeo, Imidacloprid, Beauveria bassiana, Metharizium anisopliae and Chile+garlic+soap compared to the control that was only water application. The variables evaluated were: number of nymphs, adults and winged $M$. sacchari per plant, as well as some economic variables such as the yield in $\mathrm{kg} \mathrm{ha}^{-1}$ for evaluated treatment, partial budget analysis, dominance analysis and marginal rate of return. The results obtained in the study determine that the treatments Engeo and Imidacloprid were those that presented the best control of M. sacchari, better commercial yields were obtained by the Engeo and Imidacloprid treatments, the best rates of marginal return were obtained in the Imidacloprid treatment.

Keywords: Insecticides, pest, control.
\end{abstract}




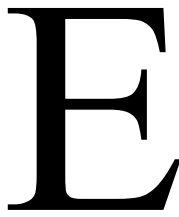

ntre los principales cultivos del mundo, el sorgo (Sorghum bicolor L. Moench) es uno de los menos conocidos por europeos y norteamericanos, en estados unidos muchos lo conocen solo como un producto para hacer jarabe, un jugo espeso y muy sabroso, similar a la melaza, que se obtiene mediante el prensado de cañas de sorgo dulce. Pero esta industria constituye apenas un uso muy limitado de este cereal. El sorgo es una fuente alimenticia muy importante para el hombre y los animales en muchos países de climas cálidos. El sorgo, es el quinto cultivo más importante entre los cereales del mundo después del trigo (Triticum sativum L.), el maíz (Zea mays L.), el arroz (Oryza sativa L.) y la cebada (Hordeum vulgare L.), (Compton, 1990). El sorgo es una fuente de calorías necesarias para el buen funcionamiento del organismo ya que contiene entre $7.10-14.20 \%$ de proteínas, $2.4-6.5 \%$ de lípidos y de 70 $-90 \%$ de carbohidratos (Hulse et al., 1980).

El sorgo es un cereal originario de la India y de la zona central África y apareció en tiempos prehistóricos hace $5000-7000$ años, en estas regiones es un alimento básico de la dieta de millones de personas, sin embargo, los países desarrollados no incluyen al sorgo en su alimentación, sino que lo emplean como forraje para el alimento de ganado. La planta de sorgo se adapta a una amplia gama de ambiente y produce granos bajo condiciones desfavorables para la mayoría de los otros cereales. Debido a su resistencia a la sequía, se considera como el cultivo más apto para las regiones áridas con lluvias erráticas (Purseglove, 1972). El cultivo es más popular en los trópicos semiáridos con una precipitación anual de más de $500 \mathrm{~mm}$, aunque su rendimiento potencial es semejante al del maíz $16500 \mathrm{~kg} \mathrm{ha}^{-1}$ (Pickett y Fredericks, 1959).

En Nicaragua el cultivo de sorgo es la base para la producción de alimentos para la nutrición de ganado bovino, porcino y aves de corral a nivel de todo el país; siendo necesario realizar investigaciones en dicho cultivo para así explotar al máximo el potencial de rendimiento en esta especie en nuestras condiciones agroecológicas (Pedroza, 1990).

La caída en el rendimiento del sorgo, según el Anuario del Banco Central, se dio en el ciclo 2015-2016, pasando de 28.7 quintales por manzana en el ciclo previo a 23.2 quintales. En el período 2016-2017 este cultivo alcanzó los 25.4 quintales por manzana. La mayoría de las áreas sembradas en Nicaragua son manejadas por grandes productores con altas tecnologías, utilizando híbridos y variedades mejoradas implementando sistemas de monocultivo durante todo el año, realizando un uso intensivo de la tierra trayendo como consecuencia agotamiento de los suelos y deterioro de los recursos existentes como el agua y la fertilidad (Alemán y Tercero, 1991).

Al menos tres millones de dólares dejarán de percibir los productores de sorgo en el actual ciclo agrícola 2016, ya que la producción se redujo en unos trescientos mil quintales, debido a que la siembra fue tardía en espera del establecimiento de la temporada lluviosa. Además, parte del área sembrada fue afectada por la plaga de pulgón amarillo (Melanaphis sacchari, Zehnter). "El factor clima es preponderante en este cultivo, por esperar las lluvias la gente sembró fuera del período óptimo, que es entre el 15 y el 31 de agosto, algunos el 15 de septiembre todavía estaban sembrando, entonces los rendimientos se cayeron y la cosecha se redujo en al menos 40 por ciento", menciono Francisco Vargas, director ejecutivo de la Asociación Nacional de Productores de Sorgo (Vargas, 2017).

En ciclos anteriores el sector había alcanzado rendimientos de entre sesenta y 62 quintales por manzana y en el ciclo actual fueron muy pocos los que alcanzaron volúmenes similares. La mayoría obtuvo entre treinta y 35 quintales por manzana. Eso redujo la cosecha que se estimaba en más de un millón de quintales a solamente unos 800000 mil quintales, que serán entregados a la industria local. Es decir, se redujo en al menos 300000 quintales que de haberse vendido a unos diez dólares por quintal habrán generado unos tres millones de dólares. Además, no se alcanzó la meta de sembrar las 35000 manzanas de sorgo rojo industrial que contempla el Plan Nacional de Producción, Consumo y Comercio para el ciclo agrícola 2016-2017. La semilla disponible solo permitió sembrar unas 22000 manzanas, de las cuales unas trescientas se perdieron por la plaga de pulgón amarillo (Vargas, 2017).

"La realidad es que la proyección era sembrar 35000 manzanas y si el invierno se hubiera visto notablemente normal se habría conseguido semilla para sembrar más, pero la gente prefirió abandonar un poco el sorgo y probar con otros cultivos que tenían mejor precio entre ellos el maní y la caña", sostiene (Vargas, 2017). Adicionalmente la situación se agravó porque el precio del sorgo local tiene que competir con el del maíz amarillo que se produce en Estados Unidos y que además de recibir subsidio, entra al país sin pagar arancel. "Entonces, la gran industria avícola local que compra nuestra cosecha para alimentar a las aves prefiere importar ese maíz que es más barato" (Vargas, 2017). 
Vargas en 2017, propone que el sorgo solo se podrá seguir produciendo si se consiguen otros nichos de mercado, entre ellos la elaboración de alimento para ganado y cerdos o el abastecimiento a los pequeños avicultores; así como almacenar la cosecha para esperar mejores precios, ya sea en infraestructura propia o comprando dicho servicio a la Empresa Nicaragüense de Alimentos Básicos (ENABAS). En el caso de que se revise el Tratado de Libre Comercio con Estados Unidos el sorgo sería beneficiado ya que el maíz amarillo tendría que pagar impuestos para su ingreso al país (Vargas, 2017).

El origen del pulgón amarillo se localiza en África y actualmente se encuentra en diversos países: Asia, Australia, el Caribe, Centro y Sudamérica. Se desconoce cómo llegó a todas estas áreas, sin embargo, se especula que este biotipo de pulgón de la caña de azúcar resultó de un cambio genético en la población estadounidense existente o de una nueva introducción a los Estados Unidos. El Ingeniero mexicano y experto en sorgo Noé Montes en 2016, menciona que en Nicaragua se puede propagar la plaga del pulgón amarillo del sorgo debido a que todos los materiales genéticos que existen en el país poseen diversos grados de susceptibilidad (Montes, 2016).

El pulgón amarillo del sorgo es considerado una de las plagas más dañinas para el cultivo de sorgo, llegó a México en 2013 y ha ocasionado graves daños a la producción de este cultivo en varios estados. En Guanajuato, en el 2015 esta plaga afectó la producción de sorgo tanto en riego como en temporal, reduciendo la producción hasta en un $100 \%$ en los sitios donde no se atendió el problema (Montes, 2016).

Esta investigación se justifica ya, que en Nicaragua ninguna institución del sector o ninguna persona o científico nacional ha realizado estudios con el pulgón amarillo del sorgo. El pulgón amarillo del sorgo causó pérdidas en el cultivo del sorgo alrededor del $35-40 \%$ en la postrera del ciclo del sorgo 2016, el sector perdió unos 10 millones de dólares, lo que significo unos 300000 quintales menos ese año (Álvarez, 2016). Este año la mayoría de los productores de sorgo en este ciclo 2016, produjeron como promedio 35 quintales por manzana, anteriormente ellos producían entre 60 y 65 quintales por manzana (Álvarez, 2016).

Los productores del sorgo en el país han solicitado públicamente que instituciones como la UNA, que es una institución donde se realiza investigación y sus investigadores realicen estudios de alternativas de manejo. Los productores de sorgo no fueron capaces de controlar esta pla- ga en el ciclo pasado, el método de control lo enfatizaron en el uso de insecticidas químicos sintéticos, y aun así fue difícil el manejo de esta plaga, los productores nos sugirieron hacer un estudio de búsqueda de alternativas de manejo de la plaga, probando opciones botánicas, biológicas y químicas también, los resultados de esta investigación serán un gran aporte al conocimiento científico nacional, además de dar respuesta a una problemática tan sentida por los productores de sorgo a nivel nacional. El objetivo de este estudio es contribuir al conocimiento científico nacional a través de la evaluación de insecticidas de origen químico, biológico y botánico para el manejo del pulgón amarillo del sorgo.

\section{MATERIALES Y MÉTODOS}

Ubicación del área de estudio. El estudio se realizó a partir del mes de julio del 2017 y concluyó en diciembre del mismo año, el estudio se estableció en la finca el plantel propiedad de la Universidad Nacional Agraria, localizado en el kilómetro 30 Carretera, Tipitapa-Masaya. Corresponde a una zona que se considera como bosque seco tropical, se ubica entre las coordenadas geográficas $12^{\circ} 06^{\prime} 24$ " de Latitud Norte y entre los $86^{\circ} 04^{\prime} 06^{\prime \prime}$ de Longitud Oeste. Se encuentra a una altura de 65 metros sobre el nivel del mar (msnm), con temperatura promedio de $28{ }^{\circ} \mathrm{C}$, la precipitación promedio anual oscila entre los 796-800 mm, con humedad relativa de $71 \%$ y viento con velocidad de $3.5 \mathrm{~m} / \mathrm{s}$ (INETER, 2009).

Diseño experimental. El estudio se estableció como un experimento en diseño de bloques completos al azar (BCA) con cuatro repeticiones y seis tratamientos. El tamaño de cada parcela correspondió a $4 \mathrm{~m}$ de largo por 5 $\mathrm{m}$ de ancho con un área de $20 \mathrm{~m}^{2}$ por cada tratamiento, formando un área de $120 \mathrm{~m}^{2}$ por bloque, siendo el área total del ensayo de $720 \mathrm{~m}^{2}$ (ver Anexo 6. Plano de campo). La distancia de siembra utilizada fue de $1 \mathrm{~m}$ entre surco y $0.8 \mathrm{~m}$ entre planta, dejando 13 plantas por metro lineal, 52 plantas por surco, siendo un total de 1040 plantas en todo el experimento.

Muestreo de insectos. Para determinar el momento de aplicación de cada uno de los tratamientos, los muestreos se realizaron semanalmente, desde los $15 \mathrm{dds}$, hasta la cosecha, por la mañana y de forma directa, donde se muestreó ninfas, adultos y alados de pulgón amarillo. Para la obtención de los datos se seleccionaron 5 puntos al azar por parcela, en cada punto se tomaron 5 plantas, para un total de 25 plantas muestreadas por parcela y 600 plantas 
en todo el experimento; se muestreó específicamente el envés de la hoja y el tallo de la planta.

Aplicaciones de insecticidas. Las aplicaciones de los productos se realizaron en base a los datos obtenidos en el muestreo, utilizando un nivel crítico poblacional de un pulgón por planta de sorgo (Trabanino, 1997). Como parámetro de decisión para aplicar el tratamiento. Las aplicaciones se realizaron por aspersión directa al envés de la hoja haciendo uso de bomba de mochila con capacidad de 20 litros de agua y se efectuaron por las tardes para evitar deriva del producto.

\section{Descripción de los tratamientos}

Tratamiento 1. Engeo ${ }^{\circ}$ (Tiametoxam $12.62 \% \quad$ y lambda-cyalotrina 9.49\%): Engeo posee acción de contacto y tiene propiedades sistémicas, la dosis utilizada será de $3 \mathrm{cc}$ por litro de agua. Es un insecticida del grupo neonicotinoides y actúa sobre el sistema nervioso de los insectos en la pos-sinapsis interfiriendo los receptores de acetilcolina. (Lanuza y Rizo, 2012).

Tratamiento 2. Imidacloprid ${ }^{\circledR}$ : Es un insecticida sistémico que actúa como neurotóxinas para insectos. Este producto agroquímico pertenece al grupo de químicos llamados neonicotinoides que afectan en el sistema nervioso central de los insectos, la dosis utilizada será de $5 \mathrm{cc}$ por litro de agua (Lanuza y Rizo, 2012).

Tratamiento 3. Beauveria bassiana, Bassi. Cepa 114: este hongo pertenece a la clase Deuteromycetes, orden Moniliales, familia Moniliaceae (Barnet y Hunter, 1972). En general las fases que desarrollan los hongos sobre sus hospedantes son: germinación, formación de apresorios, formación de estructuras de penetración, colonización y reproducción. El inoculo o unidad infectiva está constituida por las estructuras de reproducción sexual y asexual, es decir, las esporas y conidias. La dosis es de 200-300 gr/mz. Monzón (2001) menciona que la cepa 114 del hongo $B$. bassiana, actúa contra varias especies de insectos de las familias curculionidae y en otras familias dentro del orden coleóptera y áfidos.

Tratamiento 4. Metharizium anisopliae, Metschnikoff. Cepa 114: M. anisopliae (Metschnikoff). Este patógeno se encuentra ampliamente distribuido en la naturaleza, siendo aislado fácilmente de suelos, donde puede sobrevivir por lapsos prolongados, también ha sido aislado de una gran variedad de insectos, siendo utilizados en programa de control de plagas a nivel mundial. Ataca naturalmente más de 300 especies de insectos de diversos órdenes, algunas plagas que son afectadas por este hongo son la salivita de la caña de azúcar (Aeneolamia contigua, Walker), y chinches plagas de diversos cultivos. Los insectos muertos por este hongo son cubiertos completamente por micelio, el cual inicialmente es de color blanco, pero se torna verde cuando el hongo esporula (Monzón, 2001). La dosis es de 200-300 gr/mz. Monzón (2001) menciona que la cepa 114 del hongo $M$. anisopliae, actúa contra varias especies de insectos de la familia curculionidae y en otras familias dentro del orden coleóptera y áfidos.

Tratamiento 5. Chile (Capsicum annuum, L) Fam. Solanáceas + Ajo (Allium sativum L.) + detergente (xedex). El chile contiene una sustancia de pungencia llamada capsicina que al ser aplicada sobre los insectos plagas genera una sensación de ardor en todo su cuerpo, por lo cual los insectos dejan de alimentarse, huyen del lugar y mueren. (Jiménez-Martínez y Varela, 2013). El ajo contiene compuestos de azufre (tiosulfatos) los cuales sobre excitan el sistema nervioso de los insectos y ácaros produciendo irritación, desorientación y repelencia (Jiménez-Martínez y Varela, 2013). El detergente actúa como adherente al follaje, cuando entra en contacto con los tejidos grasos de ácaros los mata por deshidratación (Martínez y Jirón, 2011), la dosis utilizada fue de 100 gramos de chile, 28 gramos de detergente y una cabeza de ajo, molido y disuelto en un litro de agua, con 24 horas de reposo, aplicar con bomba de chile de 20 litros (JiménezMartínez y Varela, 2013).

Tratamiento 6. Testigo, en este tratamiento se aplicó solamente agua.

Variables evaluadas. Las variables se tomaron con una hoja de muestreo diseñada por nosotros desde los 7 días después de la siembra, realizando monitoreo una vez por semana hasta la cosecha, donde se muestreaban todas las partes de la planta específicamente el envés de la hoja, lugar donde se encuentra más frecuente el pulgón.

Número de ninfas de $M$. sacchari. se muestreó la parte del envés de la hoja donde se muestrearon 25 plantas por tratamientos, dando un total de 100 plantas muestreadas en todo el experimento. 
Número de adultos de $M$. sacchari. se muestreó la parte del envés de la hoja donde se muestrearon 25 plantas por tratamientos, dando un total de 100 plantas muestreadas en todo el experimento.

Número de alados de M. sacchari. se muestreó la parte del envés de la hoja donde se muestrearon 25 plantas por tratamientos, dando un total de 100 plantas muestreadas en todo el experimento.

Rendimiento del sorgo en $\mathbf{k g ~ h a}^{-1}$. Para obtener los datos de rendimiento por hectárea se realizó una cosecha por cada tratamiento evaluado en el estudio al finalizar el ensayo. Para obtener los datos de rendimiento por hectárea se efectuó un solo corte a los 120 dds, donde se cosecharon todas las panojas de las plantas por cada uno de los tratamientos, se pesó el total de cada tratamiento evaluado, para obtener el peso en $\mathrm{kg} \mathrm{ha}^{-1}$.

Análisis económico. Se realizó un análisis económico de presupuesto parcial, análisis de dominancia y tasa de retorno marginal, siguiendo la metodología propuesta por el CIMMYT (1988), la que considera diferentes costos, rendimientos y beneficios.

Análisis de los datos. Una vez recolectados los datos en campo se ordenaron por variable y por tratamiento para luego realizar un análisis de varianza ANDEVA, PROC GLM en SAS, V.9.1, (SAS, 2003). Se realizó una separación de medias por Duncan $(P=0.05)$.

\section{RESULTADOS Y DISCUSIÓN}

Fluctuación poblacional de alados de $M$. sacchari. Se midió la fluctuación poblacional de alados del pulgón amarillo del sorgo en los diferentes tratamientos evaluados (Figura 1). Se encontró que las poblaciones de alados de este insecto se encontraron en todas las fechas de muestreo, los mayores picos poblacionales se presentaron en las fechas septiembre $1 \mathrm{y}$ septiembre 8 , estas poblaciones fueron mayores en los tratamientos testigo seguido del tratamiento Chile + ajo + detergente y M. anisopliae. El análisis de varianza realizado a los tratamientos evaluados nos muestra que existe diferencia significativa entre los tratamientos $(P$ $=0.6369)$, donde el menor número de alados del pulgón amarillo fue encontrado en Engeo, seguido de Imidacloprid y $B$. bassiana, comparados con el testigo (Cuadro 1).

Se observó que Engeo fue el tratamiento que mejor control tuvo sobre los tres estados del pulgón amarillo evaluados en nuestro estudio. La reproducción de $M$. $s a$ cchari es predominantemente asexual con hembras adulteras ápteras y aladas que dan origen a ninfas (Voegtlin et al., 2003). (Rayo y Mena, 2015) mencionan que los insecticidas de origen botánico tuvieron efecto contra insectos chupadores como mosca blanca en el cultivo de tomate, siendo Chile+detergente el insecticida que mejor control presento en su estudio.

(Rodríguez y Terán, 2014) mencionan que los insecticidas químicos como Imidacloprid a una dosis de 105 gramos de ingrediente activo (ia) por $\mathrm{ha}^{-1}$, Sulfoxalor a una dosis de $12 \mathrm{gr}$ de (ia) por ha ${ }^{-1}$, metamidofos a una dosis de 900 gr son excelentes para el control de M. sacchari.

El pulgón se alimenta de la savia que la planta necesita para crecer desarrollarse y formar granos. Las infestaciones severas de pulgón causan que las hojas se cubran con una sustancia pegajosa y brillante llamada mielecilla la cual favorece al crecimiento de un hongo llamado fumagina de color negro, que cubre toda la hoja lo cual provoca que se sequen y se mueran, afectando la formación de los granos de la panoja (CESAVEG, 2010).

Existen diversos factores naturales de orden climático y biológico que afectan las poblaciones de áfidos. Entre los factores climáticos que afectan el desarrollo de poblaciones se encuentran los cambios repentinos de temperatura, niveles altos de humedad atmosférica y precipitación, en relación con los factores biológicos existen insectos depredadores de áfidos principalmente de las familias Coccinelidae (Dorestes, 1998).

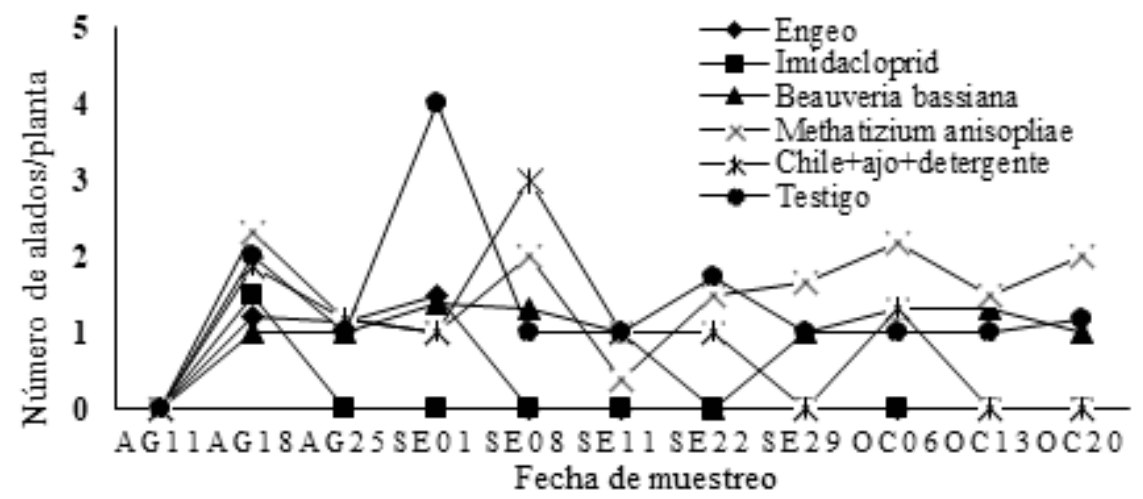

Figura 1. Fluctuación poblacional de alados de M. sacchari. en los tratamientos evaluados. 
Cuadro 1. Análisis de varianza de las poblaciones de ninfas, adultos y alados de pulgón amarillo por tratamiento evaluado

\begin{tabular}{lccc}
\hline & $\begin{array}{c}\text { Promedio de ninfas } \\
\text { por planta } \\
\text { Media } \pm \mathrm{ES}\end{array}$ & $\begin{array}{c}\text { Promedio de adultos por } \\
\text { planta } \\
\text { Tratamientos }\end{array}$ & $\begin{array}{c}\text { Promedio de } \\
\text { alados por planta } \\
\text { Media } \pm \mathrm{ES}\end{array}$ \\
\hline Engeo & $23.46 \pm 20.49 \mathrm{a}$ & $2.48 \pm 16.55 \mathrm{a}$ & $0.26 \pm 4.17 \mathrm{a}$ \\
Imidacloprid & $99.95 \pm 21.65 \mathrm{a}$ & $79.37 \pm 17.59 \mathrm{~b}$ & $1.40 \pm 6.96 \mathrm{a}$ \\
Beauveria bassiana & $111.17 \pm 37.14 \mathrm{~b}$ & $90.53 \pm 16.94 \mathrm{c}$ & $1.77 \pm 4.10 \mathrm{~b}$ \\
Chile+ajo+ detergente & $118.93 \pm 38.36 \mathrm{c}$ & $92.53 \pm 38.25 \mathrm{~d}$ & $3.60 \pm 5.12 \mathrm{c}$ \\
Metharizium anisopliae & $132.33 \pm 21.23 \mathrm{~d}$ & $90.84 \pm 17.43 \mathrm{c}$ & $3.10 \pm 3.95 \mathrm{c}$ \\
Testigo & $135.22 \pm 21.03 \mathrm{e}$ & $93.80 \pm 35.48 \mathrm{~d}$ & $3.86 \pm 4.40 \mathrm{~d}$ \\
$\mathrm{~N}$ & 1305.00 & 1177.00 & 183.00 \\
\hline CV & 114.90 & 142.54 & 67.98 \\
\hline (F; df; P) & $(12,44 ; 1290 ; 0.0001)$ & $(14.09 ; 1162 ; 0.0001)$ & $(0.68 ; 168 ; 0.6369)$ \\
\hline
\end{tabular}

$\mathrm{ES}=$ Error estándar; $\mathrm{CV}=$ Coeficiente de variación; $\mathrm{N}=$ Numero de datos utilizados en el análisis; F= Fisher calculado; $\mathrm{df}=$ Grados de libertad del error, $\mathrm{P}=$ Probabilidad según Tukey.
Los tratamientos evaluados en este estudio muestran que el tratamiento con mayor rendimiento en $\mathrm{kg}$ $\mathrm{ha}^{-1}$ fue el Engeo con respecto a los demás, seguido de los tratamientos Beauveria bassiana, Imidacloprid y Metharizium anisopliae. Los tratamientos Chile+ajo+detergente y Testigo fueron los que presentaron menor rendimiento.
Comparación del rendimiento total en $\mathrm{kg} \mathrm{ha}^{-1 .} \mathrm{Se}$ comparó el rendimiento total en $\mathrm{kg} \mathrm{ha}^{-1}$ de las parcelas de sorgo en los tratamientos evaluados de agosto a octubre (Figura 2). Los rendimientos totales obtenidos muestran que el tratamiento con mayor rendimiento fue la parcela tratada con Engeo con $4488.64 \mathrm{~kg} \mathrm{ha}^{-1}$. Los tratamientos evaluados con Beauveria bassiana, Imidacloprid y Metharizium anisopliae obtuvieron rendimientos de 4 261.36; 4147.73 y $4034.09 \mathrm{~kg} \mathrm{ha}^{-1}$ respectivamente, en cambio los tratamientos Chile + ajo + detergente y Testigo obtuvieron los rendimientos un poco más bajos con 3465.90 y 2897.73 $\mathrm{kg} \mathrm{ha}^{-1}$ respectivamente.

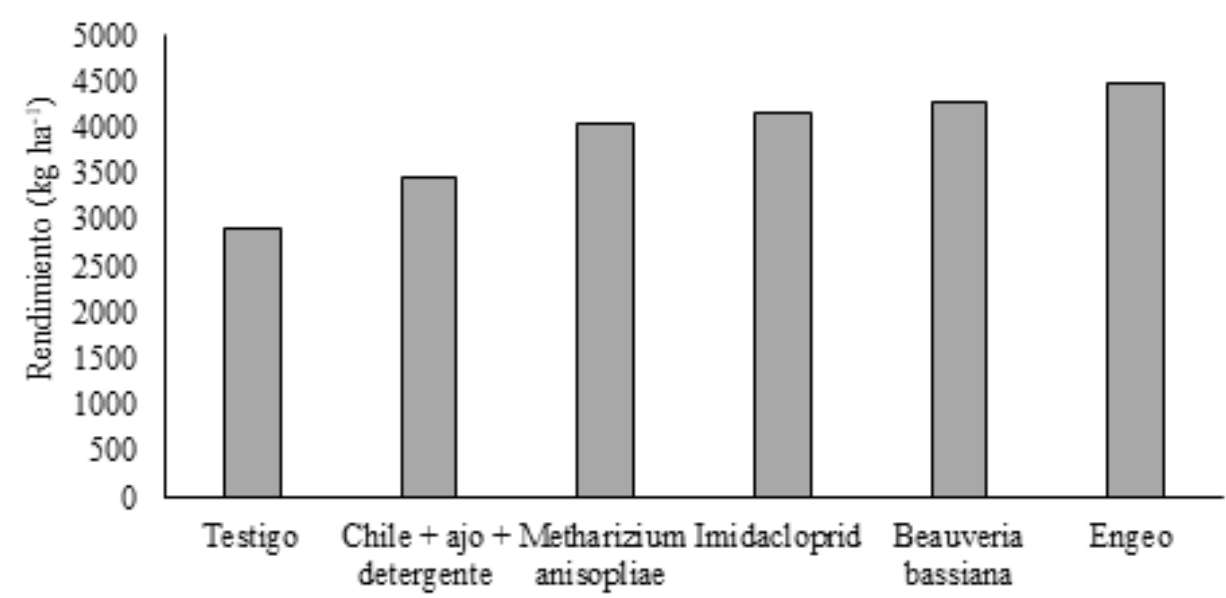

Figura 2. Comparación del rendimiento total en $\mathrm{kg} \mathrm{ha}^{-1}$ de las parcelas de sorgo por tratamientos evaluados.

\section{Comparación económica de los tratamientos para cada uno de los tratamientos evaluados}

Presupuesto parcial. EL análisis de presupuesto parcial (Cuadro 2) realizado según la metodología CIMMYT (1988) determinó que los mayores costos variables los obtuvieron los tratamientos Engeo con 190.47, Imidacloprid con 217.97 y B. bassiana y M. anisopliae con 90.47 USD $\mathrm{ha}^{-1}$, en cambio los que presentaron menores costos variables fueron Chile+ajo+detergente con 7.03 y Testigo con 5 USD ha $^{-1}$.

EL tratamiento que obtuvo el mayor beneficio neto fue el Engeo con 1366.22 USD ha-1en cambio el tratamiento que presentó los menores beneficios netos fue el Testigo con 572.33 USD ha-1.

El presupuesto parcial es un método que se utiliza para organizar los datos experimentales con el fin de obtener los costos y los beneficios de los tratamientos evaluados. En el análisis se utilizan únicamente los costos que varían de un tratamiento a otro. Por lo tanto, el proceso de aplicación de este enfoque debe generar una recomendación para los agricultores (CIMMYT, 1988). 
Cuadro 2. Presupuesto parcial para los tratamientos evaluados en el cultivo de sorgo (USD)

\begin{tabular}{|c|c|c|c|c|c|c|}
\hline Concepto & Engeo & Imidacloprid & $\begin{array}{l}\text { Beauveria bas- } \\
\text { siana }\end{array}$ & $\begin{array}{l}\text { Metharizium } \\
\text { anisopliae }\end{array}$ & $\begin{array}{c}\text { Chile+ ajo } \\
+ \text { detergente }\end{array}$ & Testigo \\
\hline Rendimiento medio $\left(\mathrm{kg} \mathrm{ha}^{-1}\right)$ & 5386.36 & 4977.27 & 5113.64 & 4840.91 & 4159.09 & 3477.27 \\
\hline Rendimiento ajustado $(10 \%)\left(\mathrm{kg} \mathrm{ha}^{-1}\right)$ & 4847.72 & 4479.54 & 4602.27 & 4356.89 & 3743.18 & 3129.55 \\
\hline Precio de campo (USD) & 0.57 & 0.57 & 0.57 & 0.57 & 0.57 & 0.57 \\
\hline Ingreso bruto (USD) & 2763.20 & 2553.33 & 2623.29 & 2483.38 & 2133.61 & 1783.84 \\
\hline \multicolumn{7}{|l|}{ Costos variables (C.V) } \\
\hline Control Químico USD ha-1 & 187.50 & 215.00 & & & & 2.03 \\
\hline Control Biológico USD ha-1 & & & 87.50 & 87.50 & & \\
\hline Control Botánico USD ha ${ }^{-1}$ & & & & & 4.06 & \\
\hline $\begin{array}{l}\text { Costo de aplicación depende del número } \\
\text { de bombadas a aplicar USD ha }{ }^{-1}\end{array}$ & 2.97 & 2.97 & 2.97 & 2.97 & 2.97 & 2.97 \\
\hline Costo total de aplicaciones USD ha- ${ }^{-1}$ & 190.47 & 217.97 & 90.47 & 90.47 & 7.03 & 5.00 \\
\hline \multicolumn{7}{|l|}{ Costos fijos en dólar } \\
\hline Depreciación de bomba de mochila/ciclo & 6.59 & 6.59 & 6.59 & 6.59 & 6.59 & 6.59 \\
\hline Depreciación de azadón/ciclo & 0.55 & 0.55 & 0.55 & 0.55 & 0.55 & 0.55 \\
\hline Depreciación de machete/ciclo & 0.30 & 0.30 & 0.30 & 0.30 & 0.30 & 0.30 \\
\hline Costo de semillas USD ha-1 & 8.57 & 8.57 & 8.57 & 8.57 & 8.57 & 8.57 \\
\hline Costo de estacas en USD ha' & 155.00 & 155.00 & 155.00 & 155.00 & 155.00 & 155.00 \\
\hline Costo de nilón USD ha ${ }^{-1}$ & 185.50 & 185.50 & 185.50 & 185.50 & 185.50 & 185.50 \\
\hline Costo total de mano de obra & 350.00 & 350.00 & 350.00 & 350.00 & 350.00 & 350.00 \\
\hline Costo de Fertilizantes & 500.00 & 500.00 & 500.00 & 500.00 & 500.00 & 500.00 \\
\hline Tasa total de Costos fijos USD ha ${ }^{-1}$ & 1206.51 & 1206.51 & 1206.51 & 1206.51 & 1206.51 & 1206.51 \\
\hline Total de Costos variables USD ha ${ }^{-1}$ & 190.47 & 217.97 & 90.47 & 90.47 & 7.03 & 5.00 \\
\hline Costo Total de producción USD ha- ${ }^{-1}$ & 1396.98 & 1424.48 & 1296.98 & 1296.98 & 1213.54 & 1211.51 \\
\hline Beneficio neto USD ha ${ }^{-1}$ & 1366.22 & 1128.85 & 1326.31 & 1186.40 & 920.07 & 572.33 \\
\hline
\end{tabular}

Precio oficial del dólar en el mes de octubre del 2017 (USD 30.60), fuente del BCN Precio del producto en campo al momento de la cosecha 0.57 USD kg.

El benéfico neto se calcula restando el ingreso bruto menos los costos totales de producción.

Análisis de dominancia. El análisis de dominancia se utiliza para los tratamientos que en términos de ganancia ofrecen la posibilidad de ser escogidos para recomendarse a los agricultores. Este análisis determina que tratamiento domina en cuanto a beneficios netos y costos variables (CIMMYT, 1998).

Cuadro 3. Análisis de dominancia

\begin{tabular}{lccl}
\hline \multicolumn{1}{c}{ Tratamiento } & $\begin{array}{c}\text { Costos Variable } \\
\text { USD ha }^{-1}\end{array}$ & $\begin{array}{c}\text { Beneficio neto } \\
\text { USD ha }\end{array}$ & Resultado \\
\hline Testigo & 5.00 & 572.33 & Dominado \\
Chile+ajo+detergente & 7.03 & 920.07 & Dominado \\
Beauveria bassiana & 90.47 & 1326.31 & No dominado \\
Metharizium anisopliae & 90.47 & 1186.40 & Dominado \\
Engeo & 190.47 & 1366.22 & No dominado \\
Imidacloprid & 217.97 & 1128.85 & No dominado \\
\hline
\end{tabular}

El análisis de dominancia realizado a este estudio (Cuadro 3), refleja que los tratamientos Testigo, Chile+ajo+jdetergente y M. anisopliae resultaron ser dominados, esto se debe a que presentan menores beneficios netos y mayores costos variables que el resto de los tratamientos incluidos en este estudio, por lo tanto, estos fueron excluidos para la realización del análisis de la tasa de retorno marginal. Los tratamientos B. bassiana, Imidacloprid y Engeo resultaron ser no dominados por lo tanto son los que se toman en cuenta para realizar el análisis de la tasa de retorno marginal.
Análisis de la tasa de retorno marginal (TRM). En análisis de retorno marginal indica lo que el agricultor puede ganar en versión promedio con su inversión cuando decide cambiar una práctica por otra más rentable, sin embargo, no se puede tomar una decisión rápida con respecto a un tratamiento sin haber determinado la tasa de retorno marginal que sería la decisión del agricultor según (CIMMYT, 1998). La tasa de retorno marginal mínima aceptable para el agricultor es de 50 y $100 \%$. 
Cuadro 4. Análisis de la tasa de retorno marginal

\begin{tabular}{lccccc}
\hline Tratamientos & $\begin{array}{c}\text { Costos Variable } \\
\text { USD ha }\end{array}$ & $\begin{array}{c}\text { Costo marginal } \\
\text { USD ha }^{-1}\end{array}$ & $\begin{array}{c}\text { Beneficio neto } \\
\text { USD ha }\end{array}$ & $\begin{array}{c}\text { Beneficio } \\
\text { marginal } \\
\text { USD ha }^{-1}\end{array}$ & $\begin{array}{c}\text { Tasa de retorno } \\
\text { marginal }^{-1}\end{array}$ \\
\hline Beauveria bassiana & 90.47 & 1326.31 & & 39.91 \\
Engeo & 190.47 & 100.00 & 1366.22 & 39.91 & 863.16 \\
Imidacloprid & 217.97 & 27.50 & 1128.85 & 237.37 & \\
\hline
\end{tabular}

En el análisis de la tasa de retorno marginal (Cuadro 4), refleja que para el control del pulgón amarillo $M$. sacchari, el mejor tratamiento es Imidacloprid ya que el productor obtiene una tasa de retorno marginal de $863.16 \%$; es decir que por cada dólar invertido el productor obtiene 8.63 dólares adicionales, por otro lado si el productor decide usar Engeo para el control del pulgón amarillo, por cada dólar invertido obtiene una tasa de retorno marginal de $39.91 \%$, lo cual equivale a 0.39 dólar adicionales por cada dólar invertido.

\section{CONCLUSIONES}

Los alados de M. sacchari comienzan a colonizar el cultivo de sorgo en los meses de agosto y septiembre.
Los tratamientos Engeo, seguido de Imidacloprid presentaron el mejor efecto de control sobre ninfas, adultos y alados de $M$. sacchari.

Los mejores rendimientos comerciales se obtuvieron en los tratamientos Engeo seguido de $B$. bassiana e Imidacloprid.

En el análisis de la tasa de retorno marginal refleja que para el control del pulgón amarillo (M. sacchari), el mejor tratamiento es Imidacloprid ya que el productor obtiene una tasa de retorno marginal d $83.16 \%$; es decir que por cada dólar invertido el productor obtiene 8.63 dólares adicionales.

\section{AGRADECIMIENTO}

Los autores de esta investigación agradecen a la Universidad Nacional Agraria (UNA) a través de los fondos concursables de la DIEP, por la financiación económica de este estudio.

\section{REFERENCIAS BIBLIOGRÁFICAS}

Alemán Zeledón, F., y Tercero Cruz, F.I. (2001). Inventario de la informacion generada en agronomía (Relaciones clima-suelo-planta-hombre) en granos basicos: arroz, maiz, sorgo y frijol en Nicaragua. Managua, Nicaragua: UNA

Alvarez, M. (12 diciembre 2016). El sorgo de Nicaragua vive su peor año. La prensa Recuperado de https://www.laprensa.com. ni/2016/12/16/economia/2151250-sorgo-nicaragua-vive-peor-ano

Barnett, H.L., y Hunter, B.B. (1972). Illustrated Genera of Imperfect Fungi. Minneapolis-Minnesota, Estados unidos: Burgess Publishing Company.

Centro Internacional de Mejoramiento de Maíz y Trigo. (1988). La formulación de recomendaciones a partir de datos agronómicos: Un manual metodológico de evaluación económico. Distrito Federal, México: CIMMYT

Comité Estatal de Sanidad Vegetal de Guanajuato. (2010). Guía para el manejo del pulgón amarillo del sorgo. Recuperado de http:// www.cesaveg.org.mx/new/manual guia\%20para el manejo de pulgon_amarillo_del_sorgo_2010.pdf

Compton, L.P. (1990). Agronomía del sorgo. San salvador, El Salvador: CENTA.

Dorestes, E. (1988). Acarología. San José, Costa Rica: IICA

Gobierno del Estado de Guanajuato. (2017). Guía para el manejo del pulgón amarillo del sorgo. Recuperado de http://www.infoagro.com/Granos Básicos/Sorgo.htm

Hulse J.H., Laing E.M., y Pearson, O.E. (1980). Sorghum and millets, their composition and nutritive value. New York, Estados unidos: Acad. press.

INETER. (2009). Registro de datos meteorológicos. Recuperado de https://servmet.ineter.gob.ni//Meteorologia/climadenicaragua.php Inter-china. (2017). Imidacloprid. Recuperado de icagrochem.com/1-28-imidacloprid/176568

Jiménez Martínez, E.S., y Rodríguez Flores, O. (2014). Insectos plagas en cultivos de Nicaragua. Managua, Nicaragua: UNA

Jiménez Martínez, E.S., y Varela Ochoa, G. (2012). Módulo práctico: Manejo Integrado de Plagas. Managua, Nicaragua: UNA

Lanuza, E; y Rizo, E. (2012). Evaluación de productos botánicos y químicos sobre el complejo mosca blanca (Bemisia tabaci Gennadius)- Geminivirus en el cultivo de tomate (Solanum esculentum, Mill.), en Tisma-Masaya. (Tesis de pregrado). Universidad Nacional Agraria. Managua, Nicaragua.

Martínez, R; y Jirón, M. (2011). Evaluación de productos botánicos y químicos para el manejo del ácaro blanco (Poliphagotarsonemus latus, Bank.) y otras plagas claves en el cultivo de chiltoma (Capsicum annuum L.) y su efecto en los enemigos naturales en Tisma, Masaya. (Tesis de pregrado). Universidad Nacional Agraria. Managua, Nicaragua.

Montes, N. (21 jul. 2016). Hemos encontrado pulgón amarrillo del sorgo en Nicaragua. El Nuevo Diario. Recuperado de http://www. elnuevodiario.com.ni/economia/409177-hemos-encontrado-pulgon-amarillo-sorgo-nicaragua/ 
Monzón, A. (2001). Producción, uso y control de calidad de hongos entomopatógenos en Nicaragua. Recuperado de https://scholar. google.com.ni/scholar?q=produccion+de+hongo+entomopatogenos + en + arroz\&hl=0\&as_sdt=0\&as_vis=1\&oi=scholart\&sa=X\&ved=0ahUKEwiSjJG3-rvVAHUE7yYKHXQuC6gQgQMIGDAA

Pedroza, H. (1990). Fundamentos de experimentación agrícola. Managua, Nicaragua: Editora de arte.

Pickett, R.C., y Fredericks, E.E. (1959). The new look in sorghum. Indianapolis, Estados unidos: Agricultural Experimental Station Report

Purseglove, J. W. (1972). I Monocotyledons. Tropical crops. 1(2): 259-286

Rayo Cruz, I.K., y Mena García, A.A. (2015). Evaluación de cinco productos botánicos para el manejo del ácaro blanco (Polyphagotarsonemus latus, Banks.) en chiltoma (Capsicum annuum L.), en Tisma, Masaya. (Tesis de pregrado). Universidad Nacional Agraria. Managua, Nicaragua.

Servicio Nacional de Sanidad Inocuidad y Calidad Agroalimentaria. (2014). Pulgón amarillo Melanaphis sacchari, Zehntner. Recuperado de https://www.gob.mx/cms/uploads/attachment/file/159533/FICHA_T_CNICA_PAS.pdf

Trabanino, R. (1997). Guía para el manejo integrado de plagas invertebradas en Honduras. El Zamorano, Honduras: Academic press.

Vargas, F. (25 febrero. 2017). El sorgo está en peligro en Nicaragua. La prensa. Recuperado de https://www.laprensa.com.ni/2017/02/25/ economia/2188762-sorgo-esta-en-peligro-en-nicaragua

Voegtlin, D., Villalobos, W., Sánchez, M., Saborió, G., y Rivera. (mayo, 2003). Áfidos alados de Costa Rica. Biología Trópical, Recuperado de https://es.scribd.com/document/127796834/Guia-de-los-afidos-alados-Homoptera-de-Costa-Rica-pdf 